\title{
DIRECT AND FLEXIBLE MESH CONTOUR COUPLING FOR AIRFOIL OPTIMISATION
}

\author{
Stephan Trautsch \\ chair of product development and construction, Otto-von-Guericke-University Magdeburg \\ Institute of Machine Design \\ 39106 Magdeburg, e-mail: stephan.trautsch@ovgu.de \\ Heike Mrech \\ professor, Merseburg University of Applied Sciences, Department of Engineering and Natural Sciences \\ 06217 Merseburg, e-mail: heike.mrech@hs-merseburg.de \\ Karl-Heinrich Grote \\ professor, chair for construction technology, Otto-von-Guericke-University Magdeburg \\ Institute of Machine Design \\ 39106 Magdeburg, e-mail: karl.grote@ovgu.de \\ Christiane Beyer \\ professor, chair of product development and design, Otto-von-Guericke-University Magdeburg \\ Institute of Machine Design \\ 39106 Magdeburg, e-mail: christiane.beyer@ovgu.de
}

\begin{abstract}
Blade profiles are used in many technical systems, including in the use of wind and water energy, and in aviation and shipping. The shape of the airfoil contour has a crucial influence on the resulting forces at the cross-section around the airfoil. The optimisation tasks are characterised by an exceptionally large number and dynamics of the influencing parameters. These are generated by a combined flowrelated and structural mechanical simulation. The current research presented here takes up these core elements of the shaping processes and makes them more flexible and transparent than conventional design methodologies. The aim is to improve the design process through a direct and flexible meshcontour coupling in such a way that the resulting shape change can be directly correlated to the initiating characteristic flow variable. The integration of the FEMSeq method enables the optimal distribution and reduction of the material without causing a failure of the overall structure.
\end{abstract}

Keywords: product development, shape optimisation, airfoil, FEMSeq

\section{Introduction}

The fundamental principles that determine the construction process of a technical product are the essential prerequisites for its area of application. In product development, a step-by-step approach enables the basic solutions for the required range of functions. These solutions are then specified in design and material specifications. (Engelmann, et al., 2021)

In the design process of turbomachinery, the design and shape optimisation of rotor blades is of particular importance. The important and difficult optimisation tasks for increasing the efficiency of the systems have to be solved here, and this is where the high design costs are usually also incurred. This concerns both the need for development and optimisation time as well as the necessity of using experts and complex optimisation methods and tools. The shape of a rotor blade has a decisive influence on the 
resulting forces on the blade profile around which the air flows and thus also on the target parameters to be optimised. These can vary greatly depending on the plant's location, whereby a multi-criteria optimisation with several target parameters is generally targeted. Decentralised energy generation requires optimal site adaptation. The type of energy carrier is irrelevant and can be realised by water as well as wind. The complex fluid-mechanical processes involved in airfoil flow and the wide variety of requirement restrictions make optimal shaping overly complicated and time-consuming. The optimisation tasks are characterised by an exceptionally large number and dynamics of influencing parameters, whereby often only an individual shaping achieves the desired optimisation effect. The present research deals with site-specific product development and in this respect specifically with the constructive shape optimisation of the blade profiles of a hydrokinetic water turbine. River systems are characterised by an overly complex flow profile. Two sites only a few meters apart can have different types of restrictions, which means that the blade profiling required differs considerably from one another. Given the global potential of these energy resources, further improvement of the site-specific shape optimisation process seems appropriate, whereby even the smallest efficiency improvements can generate enormous global added value. The aim is to improve the design process by directly and flexibly coupling the shaping external environmental influences with the internal material structure in such a way that the resulting shape changes can be placed in direct correlation with the initiating characteristic flow variables.

\section{State of the art}

Multi-objective optimisation methods are typically used in the design of rotor blades. For example, Samad (Samad, et al., 2007) uses multi-objective optimisation method based on three-dimensional Navier-Stokes's equations. Based on the four design parameters used, he generates a multi-objective solution in terms of the efficiency of the target function efficiency, total pressure and torque. A similar approach is implemented by James (James, et al., 2014). Also using a genetic multi-objective optimisation algorithm, the aim is to both maximise the annual energy production of the turbine and minimise the blade mass. Multi-objective optimisation has been shown to be extensively useful in turbomachinery design. An inflexible and one-sided optimisation strategy often generates more disadvantages than advantages, especially about the combination of strength/flow dynamics. As a result, there is a multitude of possible variants, whereby these strongly depend on each other. Without reducing one target value, it is therefore no longer possible to increase another. Artificial neural networks can accelerate this optimisation process and, under certain conditions, also contribute to increase efficiency. The combination of shape-searching genetic algorithms and time-saving artificial neural networks has thus enjoyed great attention in recent years. These also formed the basis of Verstraete (Verstraete, et al., 2011) and Uelschen`s (Uelschen, 2000) were also based on comparable approaches. Differentiations arose in the parameterisation and the formulation of the objective function and constraints. Verstraete's parameterisation of the radial blade shape was based on twenty-three design parameters. The thickness distribution of the blade's contours was not provided as an optimisation parameter. This thickness distribution was parameterised by Uelschen using two-dimensional B-spline functions and incorporated into a corresponding genetic algorithm for shape optimisation of axial blade profiles. Pierret (Pierret, 2005) concludes that a significant increase in geometric design parameters is essential for realistic blade modelling after implementing a similar approach. Notwithstanding the advantages promised by a natural-analogue optimisation method based on parameterised mathematically defined curves, there are also some fundamental problems in its use. It is assumed that "good" solutions are like each other, and 
that one can therefore arrive at better solutions step by step through mutation and recombination. Accordingly, it can be anticipated that unconventional shape variants or new design concepts are highly unlikely to be generated.

\section{Shaping with the aid of highly flexible and direct parameterisation}

The constructive design of the blade profile in the optimisation process is conventionally realised by mathematically defined curves (Bézier, B-spline, NURBS curves) and follows the premise of minimising the characteristic control points. Pierre Bézier and Paul de Casteljau laid the foundation for this at the beginning of the 1960s with the development of the Bézier curves (Bézier, 1972).

A Bézier curve is a parameterised approximation of a free-form curve. The mathematical implementation is done by multiplying so-called Bernstein polynomials, which act as a weighting function, with Cartesian control points. The control points characterise the Bézier curve and determine its geometric shape by pulling the curve towards them without touching it (Fig. 1 top). (Schiele, 2012) This approach limits both the potential spectrum of blade variants as well as the potential disruption of the velocity and pressure distribution on their design, around and on the airfoil contour. Thus, a direct link between the fluid's mechanical properties and the shape of the optimisation process cannot be achieved. This issue is a shortcoming that Pierret and Van den Braembussche recognised as early as 1999 and formulated in their paper (Pierret, et al., 1999).
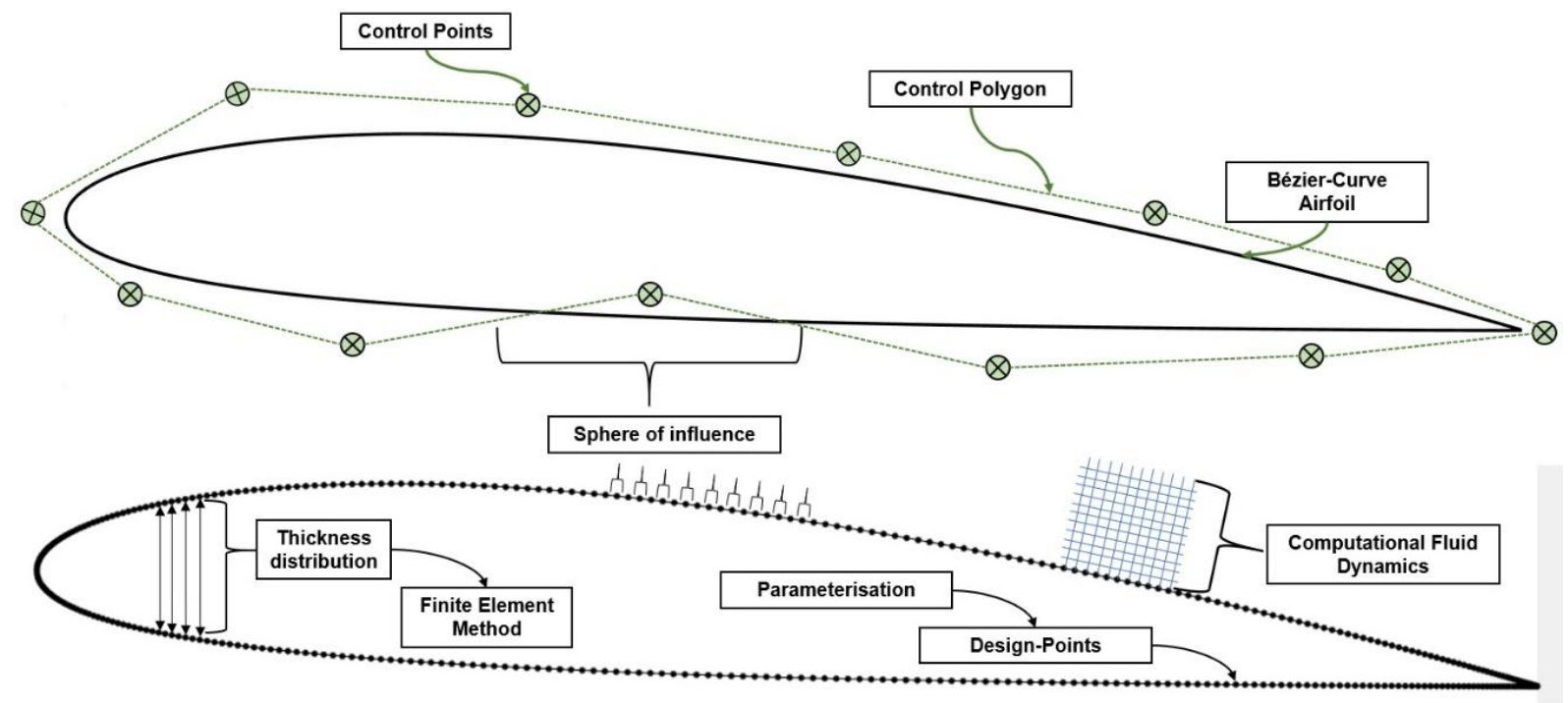

Figure 1. Schematic representation of a Bézier-Curve airfoil (top) and a direct parameterisation of a blade profile (bottom)

The research is to start with this problem. To that end, the rotor blade is first separated into twodimensional blade profiles, whereby their profile contour is parameterised by a high-dimensional point cloud (Fig. 1 bottom). The individual design points are defined by $\mathrm{x}$ and $\mathrm{y}$ coordinates. This approach achieves flexibility in shaping that allows local individual changes in the contour and an unconventional and innovative profile design. Characteristic for this parameterisation is the direct linking of the design points with the numerical fluid mechanical calculation network. The resulting pressure profile can be 
directly correlated to the geometric design of the profile contour. The sphere of influence of each individual parameter is significantly reduced and is limited exclusively to the parameterising design point itself. This procedure can act independently of neighbouring design points, and its position can be changed accordingly. Furthermore, the design point structure allows an accurate description of the existing thickness distribution of the blade profile. The difference between two corresponding design points can be used in combination with a strength and form analysis (FEM) to evaluate the structural integrity of the rotor blade. The interaction of structural and flow-mechanical analysis is indispensable for a realistic optimization process. This approach prevents geometric structures which, despite presenting a flow dynamic advantage, ultimately lack structural integrity.

\section{Sequential strength and deformation analysis}

Within the targeted shape optimisation process, a rotor blade is pressed by the fluid flow into the most advantageous geometric shape for it. As the result, the blade profile becomes narrower and adapts to the enveloping streamlines of the fluid. The numerical strength and deformation analysis should only allow this process as far as the existing material limit stresses permit. In Figure 2, the stress cross-section of a loaded rotor blade was interpolated onto a chessboard-like grid structure. Areas of higher (red) and lower (blue) material stresses are clearly visible.

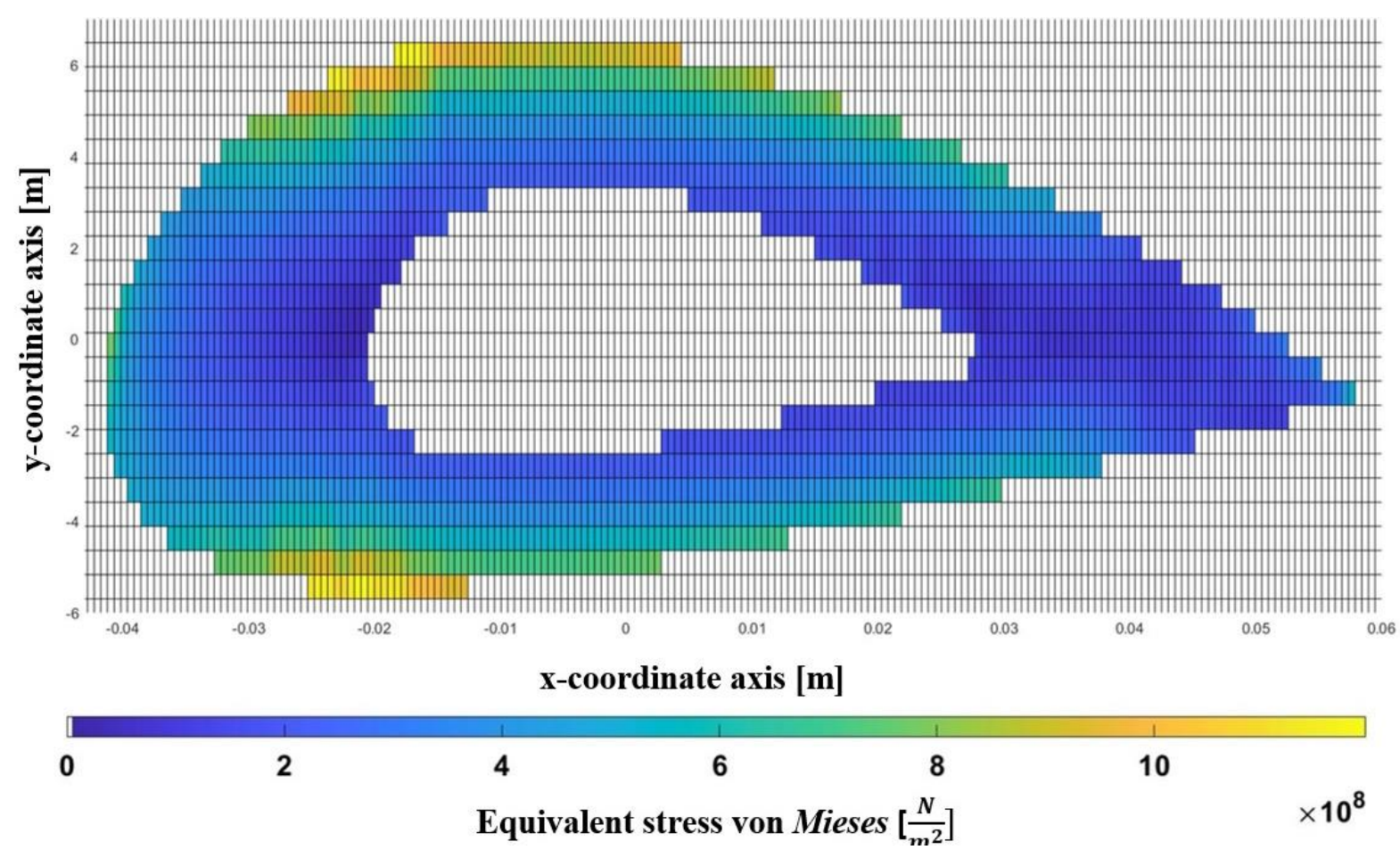

Figure 2. Interpolation of the material stresses onto a chessboard-like grid structure

A conventional strength check would declare a failure of the entire structure if the permissible maximum material parameters were exceeded globally. However, it does not make sense to "punish" the entire profile contour for the failure of a local area. Other regions are subject to significantly lower material stresses and could be further reduced or optimised in shape if necessary. The profile sections 
in which there is a high increase in material stresses are not further reduced or, under certain circumstances, strengthened again by material build-up. A sequential strength and deformation analysis (FEMSeq) allows a local stress analysis under the premise of global structural integrity. The aim is to reduce the cross-sectional area of the blade profile as much as possible by optimally distributing the material without causing a failure of the overall structure. The aim is to reduce the blade profile crosssectional area by optimally distributing the material without causing a failure of the overall structure. For this purpose, both the current geometric thickness distribution of the blade profile and the existing maximum vertical material stress within each thickness distribution are determined (Fig. 3). Within the iterative shape optimisation process, a data pool results can assign a functional relationship between the local thickness distribution and the associated vertical material stress to each FEMSeq. The iterative shape optimization process results in a data pool to which each FEMSeq can assign a functional relationship between the local thickness distribution and the associated vertical material stress. If the material-specific limit stress is used in the determined function, a minimum thickness distribution results for the considered FEMSeq with regard to the current flow restrictions. This process is carried out systematically for each individual FEMSeq. The result is a function graph for the minimum thickness distribution of the current blade profile.

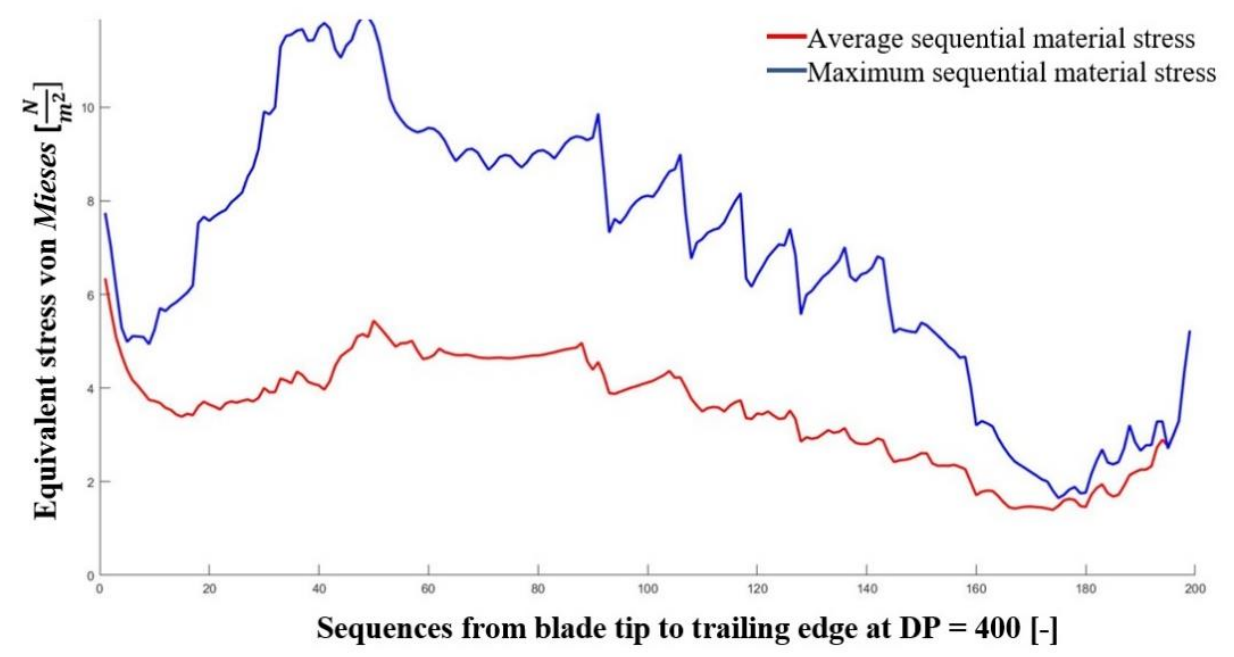

Figure 3. Average and maximum sequential strength for a blade profile with 400 design points $(N=400)$ and consequently 200 FEMSeq

Figure 4 shows the minimum thickness distribution of a blade profile. For this purpose, the thickness distribution of a NACA-2412 (Two-dimensional cross-sections of airfoil profiles for aircraft wings developed by the National Advisory Committee for Aeronautics) profile was systematically reduced by $2.5 \%$ within 25 iterations. Relevant for the shape optimisation process is the interaction between actual and minimum thickness distribution. Within the upper function graph (Fig. 4 top), the minimum thickness distribution is below the current thickness distribution of the blade profile. The current blade profile, therefore, has a larger blade cross-section available than necessary for the current blade load. A larger blade cross-section is accordingly available to the current blade profile than necessary for the current blade loading. As a result, the airfoil thickness can be further reduced without compromising the structural integrity of the blade unit. The lower function graph (Fig. 4 bottom) shows the further progression after 25 iterations. The current thickness distribution of the profile contour is almost 
completely below the determined minimum values. The structure of the blade unit would fail under these conditions. in order to counteract structural failure, the current thickness distribution of the blade profile is checked after each optimisation step using the calculated minimum value for the thickness distribution and, if necessary, corrected locally. In the regions of the blade profile with an acceptable thickness distribution, the shape optimisation is granted maximum flexibility in the design of the profile contour. Critical areas where the minimum thickness distribution could be undershot are not further reduced.

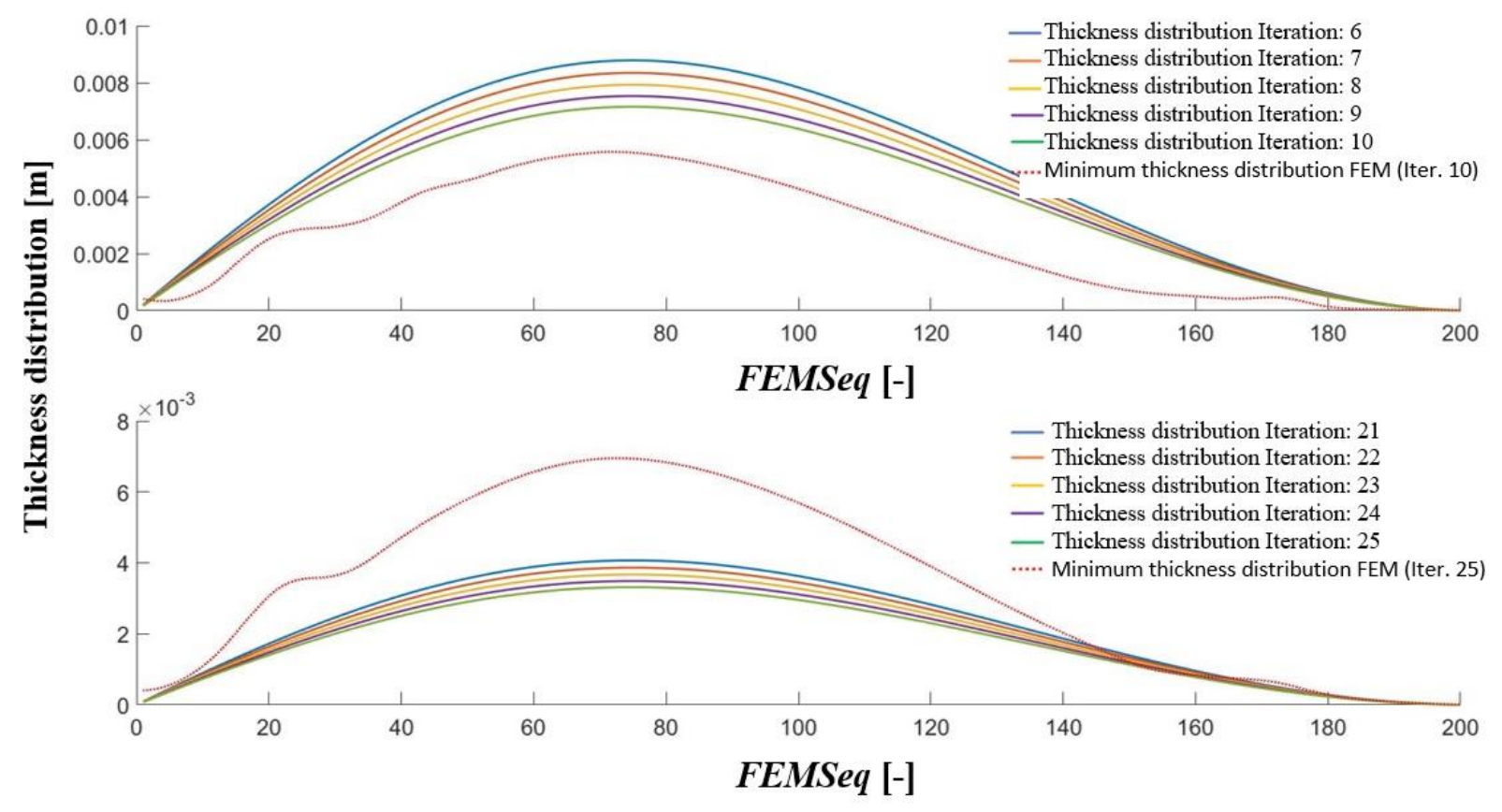

Figure 4. Minimum thickness distribution at 400 design points and consequently 200 FEMSeq

\section{Development of a shape optimisation system}

The positioning of the design points forms the foundation of the iterative shape optimisation process. Here, the profile-impacting flow variables and the resulting correlation with the profile contour are analysed and transformed into a position change of each individual design point. The pressure profile determined from the integrative numerical flow simulation at the respective design points serves as a basis. Subsequently, the resulting force vectors $\left(F_{\text {Lift }_{n}}^{i} / F_{\text {Drag }}^{i}\right)$ are calculated section by section (Fig. 4 (right)). Their sum forms the resulting force that ultimately acts on the blade profile. This can be separated into a vertical lift force $F_{\text {Lift }}^{i}$ and horizontal drag force $F_{\text {Drag }}^{i}$ (Fig. 4 (left)). The aim is to increase the lift force while reducing the drag force. At the beginning of the shape optimisation process, it is necessary to select a two-dimensional blade profile that subsequently functions as the initiating geometric starting structure. The blade profile is provided in $\mathrm{x} / \mathrm{y}$ coordinate format. The $\mathrm{y}$-coordinate of the individual design points is changed in such a way that the broadest possible spectrum of novel blade profile variants is formed. For this purpose, both a randomly acting mutation operator and a systematically operating recombination operator are used. The required geometric diversity of a new 
generation of blade profiles results from many different individuals. It seems impossible to calculate each individual pressure profile using an individual numerical fluid mechanical simulation. A too high calculation effort and an associated uneconomical time investment require an analytical alternative, which takes the form of a mathematical compensation calculation.

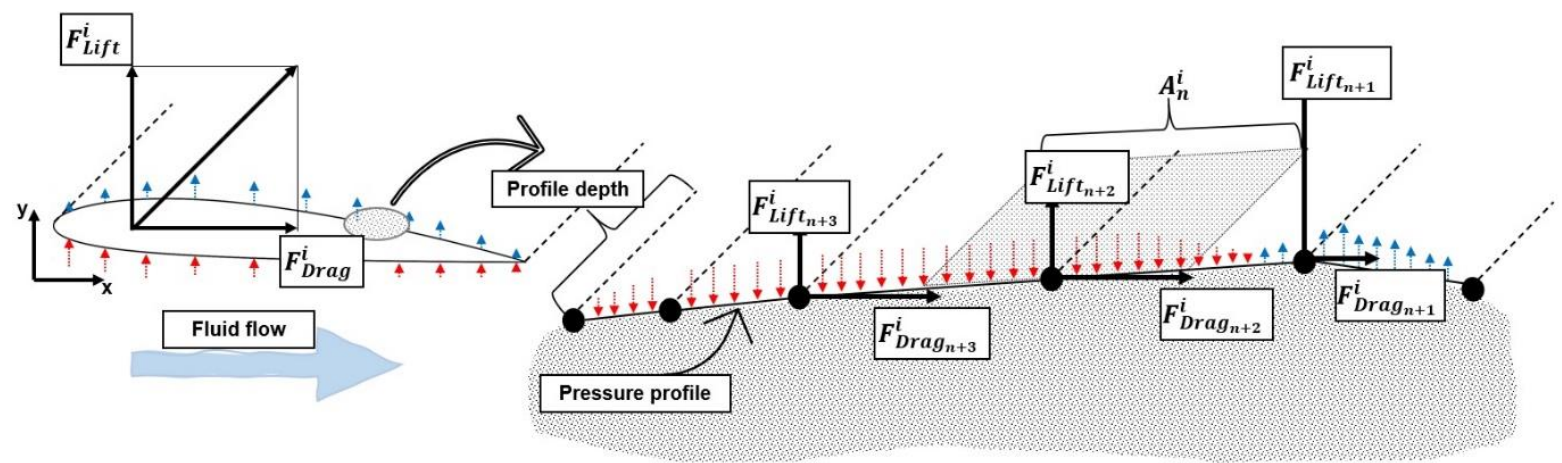

Figure 5. Lift/Drag on Airfoil (left) and Force vector on the surface (right)

The pressure value at the respective design point and the mathematical gradient of the connecting line to the design point following in the direction of flow serve as the basic variables for this compensation calculation. These calculation variables are provided separately for each design point in a database and are continuously expanded. If a new blade profile variant has been generated by the natural analogue operators' mutation and recombination, the pressure profile and subsequently the lift and drag force can be calculated by inserting the resulting mathematical slope. This process is carried out for everyone of a new generation. Finally, the potentially "best" blade profile variant is selected and verified by a numerical fluid mechanics analysis. The resulting pressure values are fed into the database and are available for the pressure profile calculation within the following optimisation iteration.

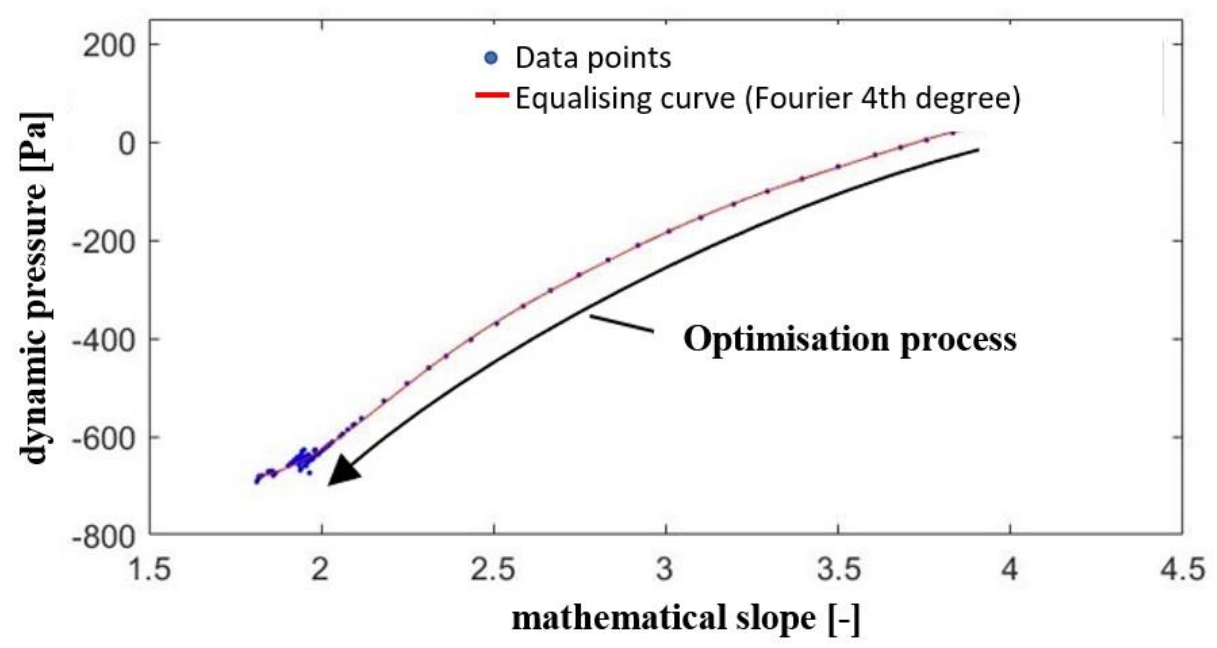

Figure 6. Balancing calculation between dynamic pressure and mathematical gradient 
All described processes were implemented in a MATLAB script and enable the automated shape optimisation of a two-dimensional blade profile with respect to the given location restrictions. Figure 7 shows the resulting structure of a fictitious location restriction. The flow medium is water, the flow velocity is $1 \frac{m}{s}$ and the angle of attack is defined as $\alpha=4,5^{\circ}$. A standardised NACA-2412 profile served as the initial geometry. The ratio of $\frac{F_{\text {Lift }}^{i}}{F_{\text {Drag }}^{i}}$ could be increased by over $32 \%$ within the shape optimisation process.

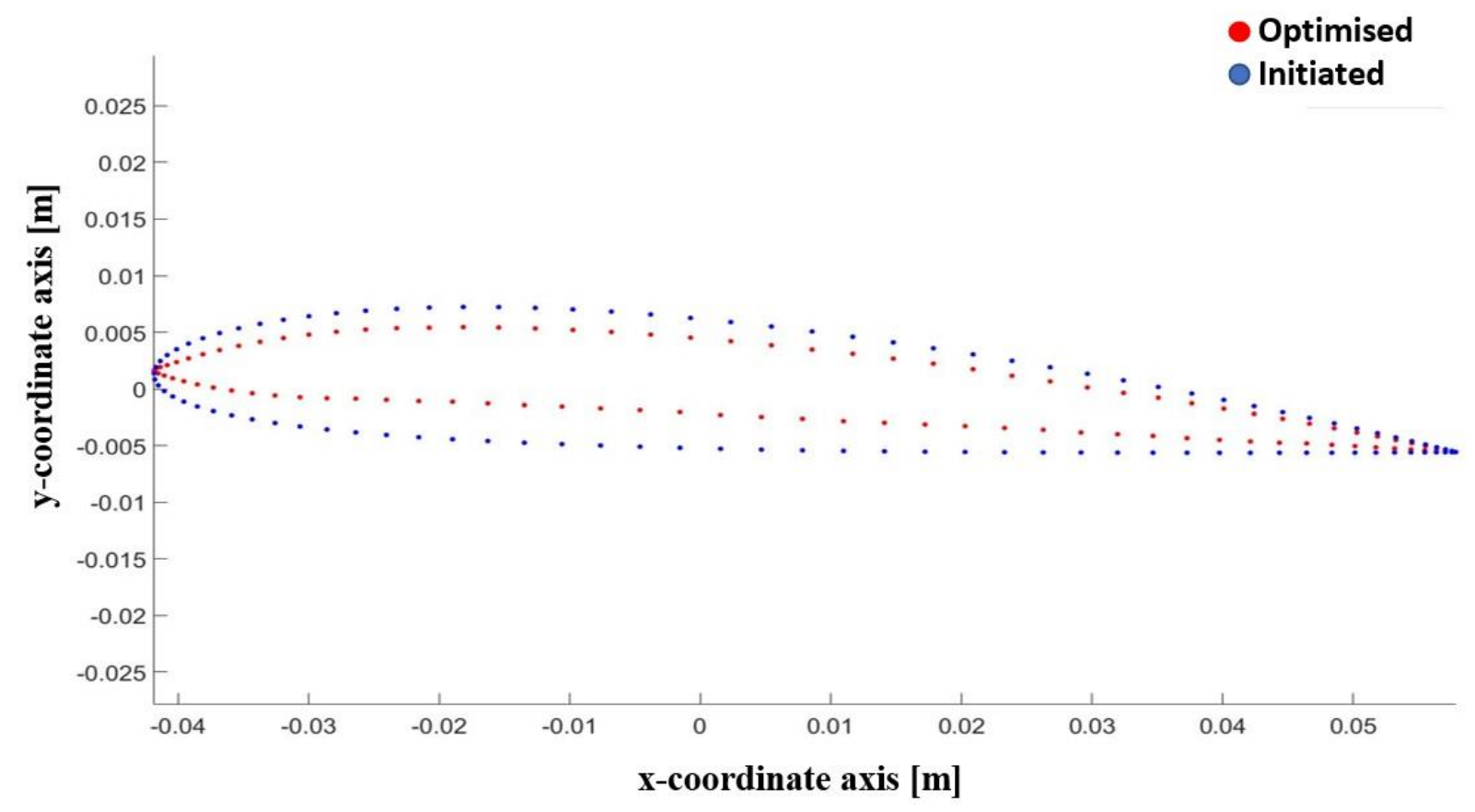

Figure 7. Comparison between initiating and optimised airfoil

\section{Conclusion}

The future challenge lies in the further technical implementation and an exact configuration of the optimisation system. With the direct and flexible mesh-contour coupling, blade profiles can be designed based on a much more precise model, with greater constructive degrees of freedom in an efficient, fast design and optimisation process. In the process, the individual character of each profile section is captured in terms of fluid mechanics and implemented in terms of shape. The integration of the FEMSeq process allows for a minimal use of material through an optimal material distribution without causing a failure of the overall structure. Possible sources of error can be seen in the two-dimensional numerical flow simulation. Physical effects that only manifest themselves in a three-dimensional flow will not be detected and therefore will not feed into the model concept. After the development of the twodimensional dynamic finite flow field, a three-dimensional expansion would also be possible in the future. 


\section{Acknowledgements}

The research reported here was carried out as part of the EFOP-3.6.1-16-2016-00011 "Younger and Renewing University - Innovative Knowledge City - Institutional development of the University of Miskolc aiming at intelligent specialization" project implemented in the framework of the Szechenyi 2020 program. The realization of this project is supported by the European Union, co-financed by the European Social Fund.

\section{References}

[1] Bézier, P. (1972). Numerical Control, Mathematics and Applications. London, Verlag John Wiley \& Sons.

[2] Engelmann, F., Breiing, A., Gutowski, T. (2021). Springer Handbook of Mechanical Engineering. [ed.] Karl-Heinrich Grote and Hamid Hefazi. s.l.: Springer International Publishing. ISBN 978-3-030-47034-0.

[3] James, A., Saijal, K. K. (2014). Multi-objective design optimization of wind. Department of Mechanical Engineering, Government Engineering College. Indien: International Journal of Engineering Research \& Technology (IJERT), 3(12), ISSN: 2278-0181.

[4] Pierret, S., Van den Braembussche, R. A. (1999). Turbomachinery blade design using a NavierStokes solver and artificial neural network. Journal of Turbomachinery, 121(2), 326-332. https://doi.org/10.1115/1.2841318

[5] Pierret, S.: Multi-objective and multi-disciplinary optimization of three-dimensional turbomachinery blades, 2005 6th World Congresses of Structural and Multidisciplinary Optimization, Rio de Janeiro, Brazil.

[6] Samad, A., Kim, K. Y., Lee, K. S.: Multi-objective optimization of a turbomaschinery blade, 2007 5th Joint ASME/JSME Fluids Engineering Conference, FEDSM2007-37434, San Diego, California, USA.

[7] Schiele, H. G. (2012). Computergrafik für Ingenieure. Berlin, Heidelberg, Springer Berlin Heidelberg. https://doi.org/10.1007/978-3-642-23843-7

[8] Uelschen, M. (2000). Entwurf und Optimierung der zweidimensionalen Gitterströmung axialer Turbomaschinenbeschaufelungen mit neuronalen Netzen und Genetischen Algorithmen. Kassel: Universität Gesamthochschule Kassel, Fachbereich Maschinenbau. ISBN: 3-933146-38-0, Dissertation.

[9] Verstraete, T., Hildebrand, A., Van den Braembussche, R. (2011). Multidisciplinary design and off-design optimization of a radial compressor for industrial application. Brüssel, Belgien, Proceedings of the 10th International Symposium on Experimental Computational Aerothermodynamics of Internal Flows. ISAIF10-153. 